Revue des patrimoines

\title{
LarvaTracing : Imagerie RMN des infestations dans les œuvres d'art en bois et matériaux organiques
}

Larva Tracing: magnetic resonance imaging on infested works of art made of wood or organic materials

Grazia Nicosia, Hervé Mathieu, Joëlle Le Roux, Anne de Wallens et Michel Dojat

\section{OpenEdition \\ Journals}

Édition électronique

URL : http://journals.openedition.org/insitu/22094

DOI : $10.4000 /$ insitu.22094

ISSN : $1630-7305$

Éditeur

Ministère de la Culture

\section{Référence électronique}

Grazia Nicosia, Hervé Mathieu, Joëlle Le Roux, Anne de Wallens et Michel Dojat, «LarvaTracing

Imagerie RMN des infestations dans les œuvres d'art en bois et matériaux organiques », In Situ [En ligne], 39 | 2019, mis en ligne le 31 mai 2019, consulté le 11 juillet 2019. URL : http://

journals.openedition.org/insitu/22094; DOI : 10.4000/insitu.22094

Ce document a été généré automatiquement le 11 juillet 2019.

\section{cc)}

In Situ Revues des patrimoines est mis à disposition selon les termes de la licence Creative Commons Attribution - Pas d'Utilisation Commerciale - Pas de Modification 4.0 International. 


\title{
LarvaTracing : Imagerie RMN des infestations dans les œuvres d'art en bois et matériaux organiques
}

\author{
Larva Tracing: magnetic resonance imaging on infested works of art made of \\ wood or organic materials
}

Grazia Nicosia, Hervé Mathieu, Joëlle Le Roux, Anne de Wallens et Michel Dojat

Les images ont été acquises sur la plateforme IRMaGE de Grenoble financée en partie par le programme français d'investissement d'avenir piloté par l'Agence nationale pour la recherche ; programme Infrastructure d'avenir en biologie santé - ANR-11-INBS-0006.

\section{Introduction}

1 Les infestations peuvent être destructives pour des biens culturels attaqués mais également pour les collections contiguës. De nombreux efforts sont réalisés dans les institutions patrimoniales, de façon précoce, pour diagnostiquer, prévenir et traiter les infestations d'insectes nuisibles. L'imagerie offre un ensemble d'outils non destructifs pour détecter la présence de larves et d'œufs. L'approche classique d'imagerie ultrasonore (l'échographie) consiste en l'émission d'une onde dans la bande de fréquences des ultrasons et la récupération d'une onde réfléchie par les objets rencontrés. Une des limitations majeures de cette technique est l'atténuation de l'onde acoustique émise par le matériel traversé et les interfaces rencontrées (par ex. air-objet). Fleming et coll. utilisent cette technique pour identifier des larves de Plectrodera scalator dans des pièces de bois ${ }^{1}$. Une larve en surface du bois a ainsi pu être repérée en réduisant la transmission de l'onde acoustique émise (fréquence : $200 \mathrm{kHz}$ ) du fait de la succession des interfaces (capteur/air, air/larve, larve/air, air/bois, bois/air, air/capteur). En revanche, ni la larve ni son mouvement n'ont pu être détectés dans la galerie creusée du fait de la trop forte atténuation du signal (interfaces bois/air et air/larve). Pour détecter la larve 
$\mathrm{du}$ foreur du bambou (Dinoderus minutus), Wanatabe et coll. utilisent le signal acoustique émis directement (fréquence: $150 \mathrm{kHz}$ ) lors des ruptures de fibres par les mandibules de la larve ${ }^{2}$. La même technique a été utilisée par Le Conte et coll. pour étudier et reconnaître automatiquement les traces sonores spécifiques, bruits et vibrations, émis par des insectes xylophages ${ }^{3}$. Elle a été appliquée à la détection des Oligomerus ptilinoides présents dans le bois en vue de la surveillance des collections d'instruments de musique. Cette approche permet d'imager des larves en activité biologique, forage et déplacement. Elle a été implémentée dans le système $\mathrm{ATAX}^{4}$ pour détecter les pics acoustiques émis lors de la rupture de fibres cellulosiques dans une bande de fréquences entre $70 \mathrm{kHZ}$ et $1 \mathrm{Mhz}$.

La micro-tomographie par rayons X permet de visualiser les larves en culture et de suivre en temps réel leur métamorphose ${ }^{5}$. Elle permet de suivre la maturation de la larve du foreur du bambou et d'estimer la consommation de bois ${ }^{6}$ avec une bonne résolution spatiale $(62 \mu \mathrm{m})$ sur un champ de vue réduit $(30 \mathrm{~mm})$. Grâce à son utilisation, couplée à des outils de traitement d'images $^{7}$, on peut estimer la densité pulpaire des grenades infestées ou non par la larve de pyrale (Thaumatotibia leucotreta).

2 Nous proposons ici d'utiliser l'imagerie par résonance magnétique (IRM) pour la détection et le suivi de larves dans les biens culturels non métalliques.

\section{Imagerie par résonance magnétique (IRM)}

3 L'IRM est une technique d'imagerie basée sur le phénomène de résonance magnétique nucléaire (RMN) ${ }^{8}$.

\section{Principe de la RMN}

4 Ce phénomène repose sur les propriétés magnétiques du noyau des atomes. En effet, un noyau possède un moment magnétique, appelé spin nucléaire, s'il possède un nombre impair de protons (le proton est une particule élémentaire de charge positive ; le noyau d'hydrogène, très répandu, est constitué d'un seul proton et possède donc un spin nucléaire). En présence d'un champ magnétique $B 0$, les protons d'un tel noyau (par exemple celui d'hydrogène associé aux molécules d'eau) vont s'aligner avec le champ magnétique (parallèlement ou antiparallèlement) et adoptent un mouvement de précession, dont la fréquence dépend du champ magnétique appliqué et du noyau considéré (cette fréquence est dans la bande des radiofréquences FM, pour l'hydrogène, par exemple, elle est de $42.58 \mathrm{MHz} / \mathrm{T}$ ). Trois étapes sont essentielles au phénomène RMN :

5 - Excitation : l'application d'une onde électromagnétique b1 à la fréquence de précession (phénomène dit de résonance) va faire basculer les protons d'un angle alpha par rapport au champ BO.

6 - Relaxation : lorsque que l'on arrête $b 1$, les protons vont relaxer, c'est-à-dire revenir à leur position originelle, alignée sur BO.

7 - Mesure du signal RMN : lors de ce retour à l'équilibre, il y a émission d'un signal radiofréquence. Sa fréquence, son amplitude et sa durée sont caractéristiques du noyau considéré et de son environnement. Le signal est mesuré par une bobine réceptrice.

8 En pratique, le champ $B 0$, de l'ordre de quelques teslas, est obtenu par un bobinage supraconducteur dans lequel circule un courant électrique de forte intensité. b1 est de l'ordre de quelques milli-teslas. L'émission de ce champ ainsi que la réception du signal 
émis sont généralement réalisées par une même bobine (ou antenne), dite alors antenne d'émission-réception. À l'origine, la RMN permet l'analyse de la structure moléculaire d'un corps physique par spectroscopie (c'est-à-dire magnetic resonance spectroscopy, MRS) : le spectre de fréquences nucléaires obtenu représentant la signature de ses constituants. L'analyse du comportement des protons soumis à un champ magnétique, et associés aux molécules d'eau largement répandues dans les corps biologiques, a ouvert la voie aux applications biologiques et médicales de la RMN. Pas moins de cinq Prix Nobel (2 en physique, 2 en chimie et 1 en physiologie et médecine) sont associés à cette technique.

\section{Principe de l'IRM}

Pour obtenir une image bidimensionnelle (2D) ou tridimensionnelle (3D), il faut dissocier les signaux provenant des différentes parties du corps à imager. Cette localisation spatiale du signal est obtenue en ajoutant un champ magnétique variant linéairement dans chaque direction d'intérêt $(\mathrm{x}, \mathrm{y})$ ou $(\mathrm{x}, \mathrm{y}, \mathrm{z})$. En effet, un champ magnétique qui varie en fonction de la position (gradient de champ magnétique) va avoir pour effet de générer des fréquences de précession des protons différentes selon leur position. C'est le principe du codage de l'espace par la fréquence. Ces différentes composantes fréquentielles seront présentes dans le signal RMN mesuré et extraites par décomposition de Fourier. La maitrise parfaite de cet encodage fréquentiel, donc de la position du signal émis par sa fréquence, permet d'attribuer à chaque partie de l'image la partie du signal qui lui correspond. L'amplitude du signal mesuré est ensuite encodée sous forme de niveau de gris.

\section{Séquences et contrastes}

10 En manipulant les paramètres de la séquence de mesure RMN, notamment le temps, les excitations successives $(\mathrm{TR}=$ temps de répétition), l'instant de réception du signal $(\mathrm{TE}=$ temps d'écho) et l'angle de bascule à l'excitation (alpha), on va favoriser une caractéristique spécifique présente dans le signal. Ainsi, le temps de relaxation (temps pour revenir à l'état stable après excitation) n'est pas le même pour le signal en provenance du bois que pour celui en provenance d'un corps biologique comme une larve par exemple. On peut ainsi favoriser l'un ou l'autre. C'est l'un des intérêts majeurs de cette technique non invasive: selon la séquence, le signal mesuré est le reflet de différents paramètres physiques ou physiologiques (en médecine, cela pourra être le débit sanguin, l'oxygénation du sang, le déplacement des molécules d'eau, le temps de relaxation de différents tissus, sains ou lésés...). Différentes perturbations peuvent introduire des artefacts dans les images. Toute imperfection de $B 0$ ou $b 1$ va affecter l'encodage fréquentiel et donc distordre l'image reconstruite à partir du signal mesuré. L'inhomogénéité structurelle au sein d'un même tissu biologique ou à l'interface entre deux types de tissus de susceptibilité magnétique différente va générer des perturbations du champ magnétique, donc des distorsions dans les images. Enfin, une cause majeure d'artéfact est le mouvement de l'objet à imager durant l'acquisition. 


\section{Matériel et méthodes}

11 Larves imagées. Nous avons considéré deux pièces de bois (fragment de chaise, voir fig. 1A) infestées par des larves de petites vrillettes (Anobium punctatum) et 8 échantillons de bois de $20 \mathrm{~mm}$ x $20 \mathrm{~mm}$ x $50 \mathrm{~mm}$ (voir fig. 1C) contenant (ou non) des larves de capricornes des maisons (Hylotrupes bajulus).

12 Acquisition IRM. Afin d'évaluer la faisabilité de la technique proposée, nous avons considéré trois imageurs (Biospec 94/20, AVIII-HD, Ettlingen, Allemagne) composés d'aimants à différents champs magnétiques $(4,7 \mathrm{~T}, 7 \mathrm{~T}$ et 9,4 $\mathrm{T})$ et différents diamètres d'ouverture (respectivement $400 \mathrm{~mm}$ et $200 \mathrm{~mm}$ pour les deux derniers) permettant d'évaluer la sensibilité de la méthode pour les différentes valeurs de BO. Une seule antenne émission-réception a été utilisée (voir fig.1B): antenne en quadrature, de diamètre interne $72 \mathrm{~mm}$, permettant l'obtention d'une taille d'image de l'ordre de $100 \mathrm{~mm}$ x $60 \mathrm{~mm}$. La répétition de la même expérience permet l'accumulation du signal et une augmentation $d u$ rapport signal sur bruit. Différentes valeurs de ce nombre d'accumulations (Nacc) ont été testées. Nous avons utilisé quatre types de séquence en écho de gradient, l'une (S1) pour visualiser les pièces de bois et éventuellement les larves et les trois autres (S2-S3-S4) pour maximiser la capacité de détection et de visualisation des larves. Les paramètres étaient les suivants :

13 ( $\mathrm{S} 1)$ séquence Flash 2D, TR/TE : $200 \mathrm{~ms} / 0,78 \mathrm{~ms}$, angle d'excitation : 40 $1 \mathrm{~mm}^{2}$, avec trois plans de coupes : axial, champ de vue : $50 \mathrm{~mm} \times 50 \mathrm{~mm}$, épaisseur de coupe : $80 \mathrm{~mm}$; sagittal et coronal, champ de vue : $80 \mathrm{~mm}$ x $50 \mathrm{~mm}$, épaisseur de coupe $50 \mathrm{~mm}$. Les images étaient obtenues en projection (2D) avec une durée de $6 \mathrm{~s}$ (Nacc:1) ou $1 \mathrm{~min} 16 \mathrm{~s}$ (Nacc : 12).

14 (S2) séquence Flash 2D, TR/TE: $3 \mathrm{~ms} / 1 \mathrm{~ms}$, angle d'excitation : $10^{\circ}$, champ de vue $80 \mathrm{~mm}$ x $35 \mathrm{~mm}$, épaisseur de coupe : $35 \mathrm{~mm}$, taille de pixel :0,54 $\mathrm{mm}$ x 0,54 mm, avec deux plans de coupe sagittal et coronal. Les images étaient obtenues en projection (2D) avec une

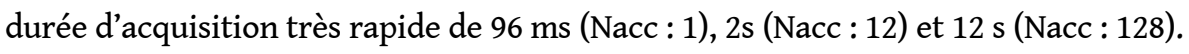

(S3) séquence Flash 3D, TR/TE : $3 \mathrm{~ms} / 0,806 \mathrm{~ms}$, angle d'excitation $10^{\circ}$, champ de vue $80 \mathrm{~mm} \times 40 \mathrm{~mm} \times 40 \mathrm{~mm}$, taille de pixel : 0,54 x 0,54 x 0,54 $\mathrm{mm}^{3}$. Les images 3D étaient obtenues avec une durée d'acquisition rapide : $2 \mathrm{~s}$ (Nacc: 1 ), $48 \mathrm{~s}$ (Nacc: 20), $10 \mathrm{~min} 14 \mathrm{~s}$ (Nacc : 256).

16 (S4) séquence Flash 3D, TR/TE : $3 \mathrm{~ms} / 0,7 \mathrm{~ms}$, angle d'excitation $10^{\circ}$, champ de vue $80 \mathrm{~mm}$ x $40 \mathrm{~mm} \times 40 \mathrm{~mm}$, taille de pixel : $0,54 \times 0,54 \times 0,54 \mathrm{~mm}^{3}$. Les images 3D étaient obtenues avec une durée d'acquisition acceptable : $9 \mathrm{~s}$ (Nacc: 1), $3 \mathrm{~min} 12 \mathrm{~s}$ (Nacc: 20), $10 \mathrm{~min} 14 \mathrm{~s}$ (Nacc: 64 ).

17 Pour notre étude, nous avons choisi de quantifier le pouvoir de détection selon les séquences utilisées par le nombre de larves détectées dans une image. La classique quantification comparative par une métrique de type rapport signal sur bruit ou rapport contraste sur bruit est dans notre cas peu adaptée du fait de la forte hétérogénéité du niveau de signal à l'intérieur du morceau de bois. 


\section{Résultats}

Nous différencions les résultats selon le type de larves, vrillettes ou capricornes à détecter.

\section{Fragment de chaise infesté par l'Anobium punctatum}

Le dispositif expérimental est indiqué sur la Figure 1 (fig. 1).

Figure 1
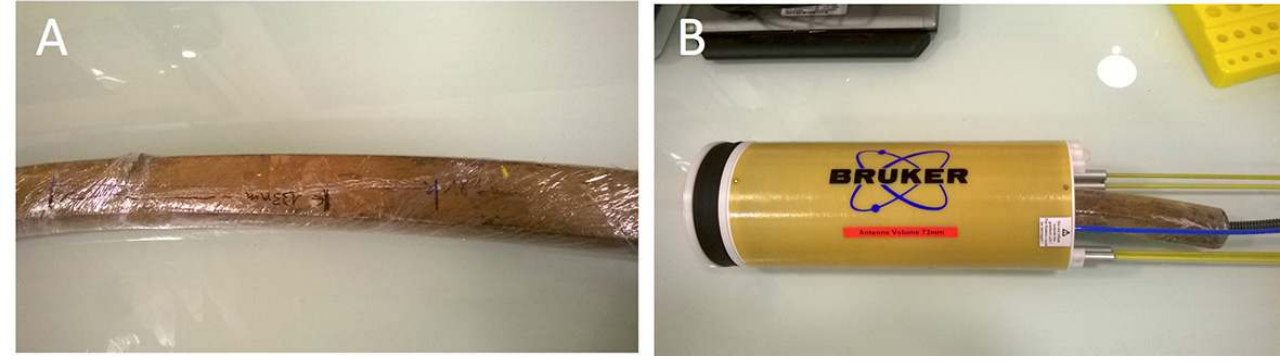

Dispositif expérimental : A : pièce de bois (fragment de chaise) infestée de petites vrillettes. B : antenne cylindrique d'émission - réception avec la pièce de bois A placée en position de mesure. (c) M. Dojat, H. Mathieu, Inserm, 2018.

Les résultats obtenus sur les différents imageurs sont résumés dans les Tables 1, 2, 3, 4 respectivement pour les séquences $\mathrm{S} 1, \mathrm{~S} 2$, S3 et $\mathrm{S} 4$.

La séquence $\mathrm{S} 1$, de type projection (une seule coupe intègre le signal de toute l'épaisseur de l'échantillon), permet la visualisation du bois et éventuellement des larves, selon leur taille et leur position (fig. 2, A-B-C). Si elle ne permet pas la détection de toutes les larves, elle est cependant utile pour le repérage du bois avec un temps d'acquisition court (6s).

Table 1

Valeurs obtenues avec la séquence S1 (FLASH 2D, résolution $1 \mathrm{~mm} \times 1 \mathrm{~mm}$ ). Nacc: Nombre d'accumulations de la même séquence.

\begin{tabular}{|l|l|l|l|}
\hline & \multicolumn{2}{|l|}{ Imageur } \\
\hline & $4,7 \mathrm{~T}$ & $7 \mathrm{~T}$ & $9,4 \mathrm{~T}$ \\
\hline Nacc (durée) & \multicolumn{3}{|l|}{ Nombre de larves détectées } \\
\hline $1(6 \mathrm{~s})$ & $3-4$ & 4 & 4 \\
\hline $12(1 \mathrm{~min} 16 \mathrm{~s})$ & $3-4$ & 4 & 4 \\
\hline
\end{tabular}


Figure 2

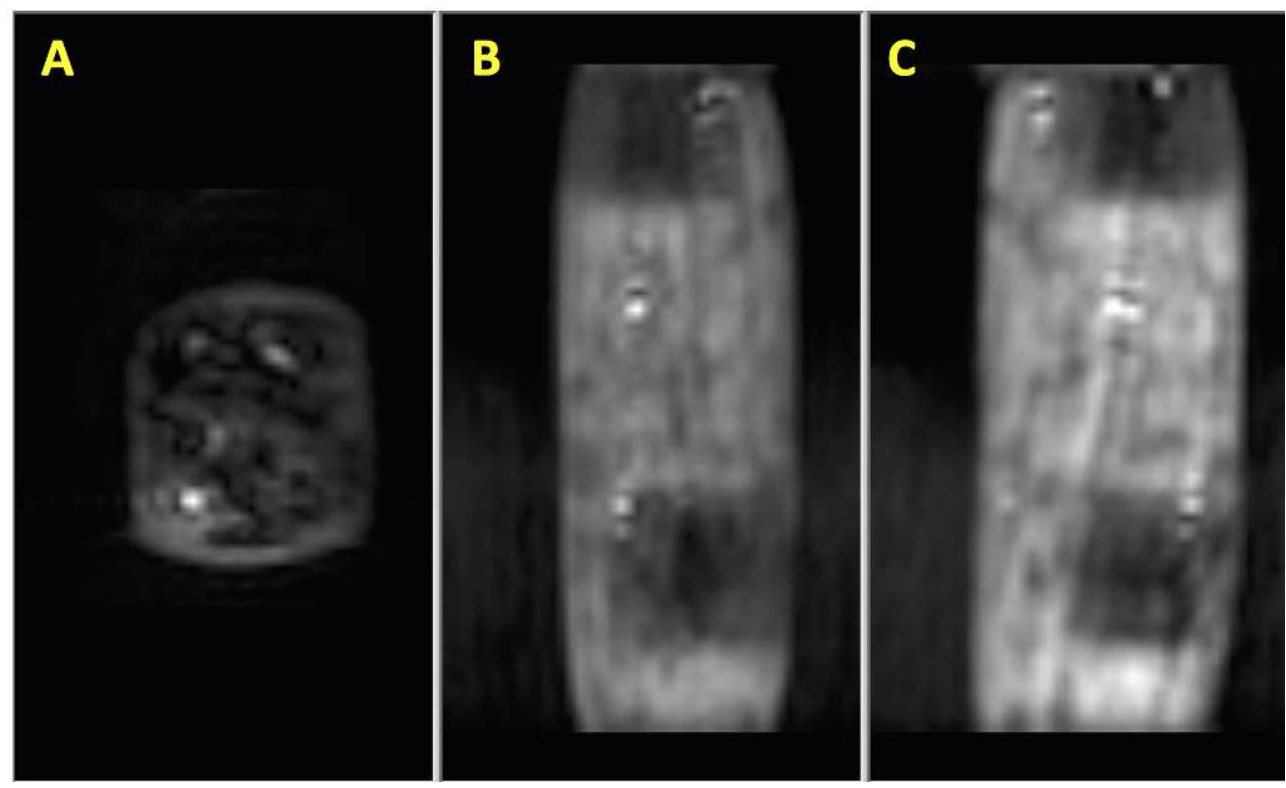

Images 2D obtenues à 4,7 T avec la séquence FLASH 2D (S1, Nacc = 1, durée $6 \mathrm{~s})$. A : projection axiale ; $\mathrm{B}$ : projection coronale $; \mathrm{C}:$ projection sagittale. Nacc $=$ Nombre d'accumulations de la même séquence.

(c) M. Dojat, H. Mathieu, Inserm, 2018

On remarquera que l'utilisation de valeurs de champ B0 différentes apporte des contrastes différents (fig. 3). Pour cette séquence, un champ B0 plus important permet une meilleure détection des larves (Figure 3C), Cependant, l'utilisation en tant que séquence de repérage est satisfaisante à $4.7 \mathrm{~T}$, même avec une durée de $6 \mathrm{~s}(\mathrm{Nacc}=1)$.

Figure 3

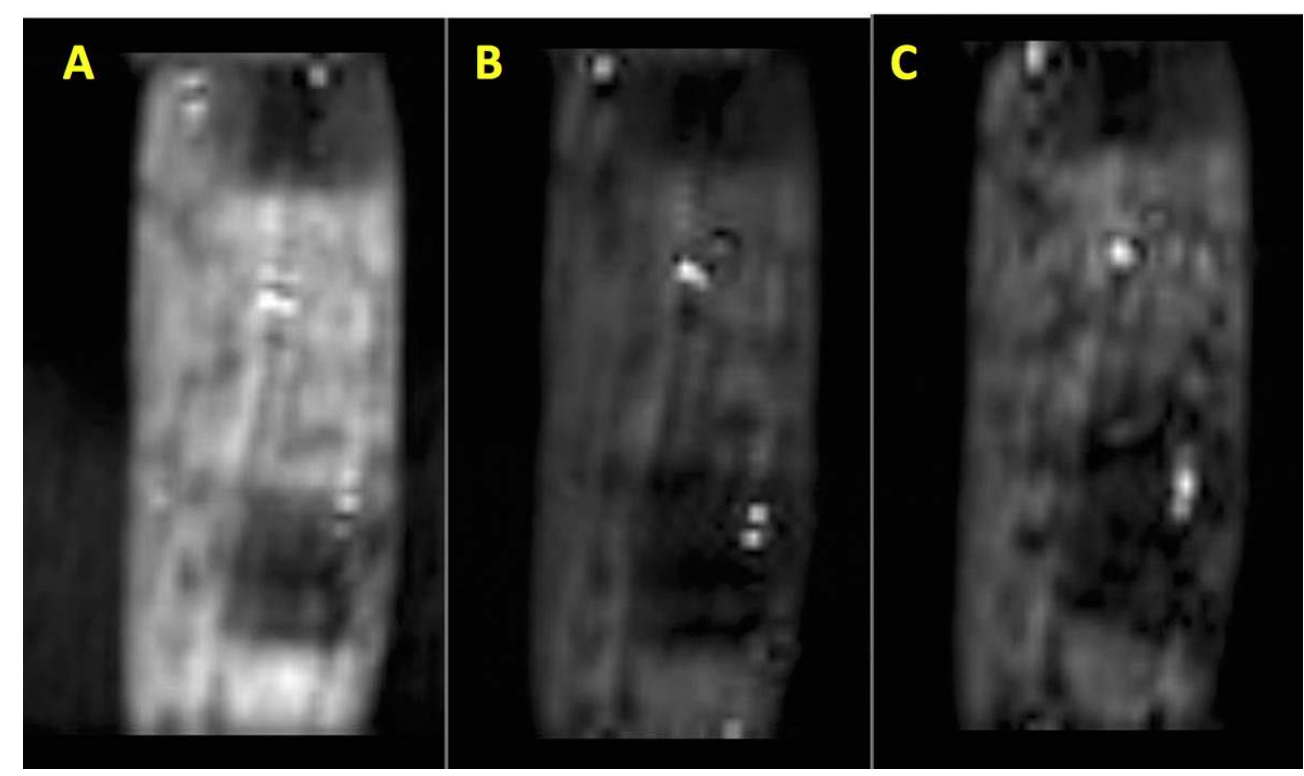

Comparaison d'images obtenues à différentes valeurs de champ B0 avec la séquence $\mathrm{S} 1$. Projections sagittales ( $\mathrm{Nacc}=12$, durée $1 \mathrm{~min} 16 \mathrm{~s}) \mathrm{A}: 4,7 \mathrm{~T} ; \mathrm{B}: 7 \mathrm{~T} ; \mathrm{C}: 9,4 \mathrm{~T}$. Nacc $=$ Nombre d'accumulations de la même séquence.

(c) M. Dojat, H. Mathieu, Inserm, 2018. 
La séquence S2 (fig. 4) permet une détection rapide et fiable de la présence de larves en un temps très court (12 s). Comme c'est une image de type projection, elle ne permet pas de différencier des larves alignées dans une même projection, et donc leur comptage (voir les incertitudes de comptage Table 2).

\section{Table 2}

Valeurs obtenues avec la séquence S2 (FLASH 2D, résolution 0,54 $\mathrm{mm}$ x 0,54 mm). Nacc: Nombre d'accumulations de la même séquence.

\begin{tabular}{|c|c|c|c|}
\hline & \multicolumn{3}{|c|}{ Imageur } \\
\hline & $4,7 \mathrm{~T}$ & $7 \mathrm{~T}$ & $9,4 \mathrm{~T}$ \\
\hline Nacc (durée) & \multicolumn{3}{|c|}{ Nombre de larves détectées } \\
\hline 1 (96 ms) & $8-16^{*}$ & $2-5 *$ & $5-8 *$ \\
\hline $12(2 \mathrm{~ms})$ & 6 & $2-3 *$ & 6 \\
\hline $128(12 \mathrm{~s})$ & 6 & 4 & 6 \\
\hline
\end{tabular}

* Présence d'artefacts pouvant être interprétés comme des larves

Figure 4

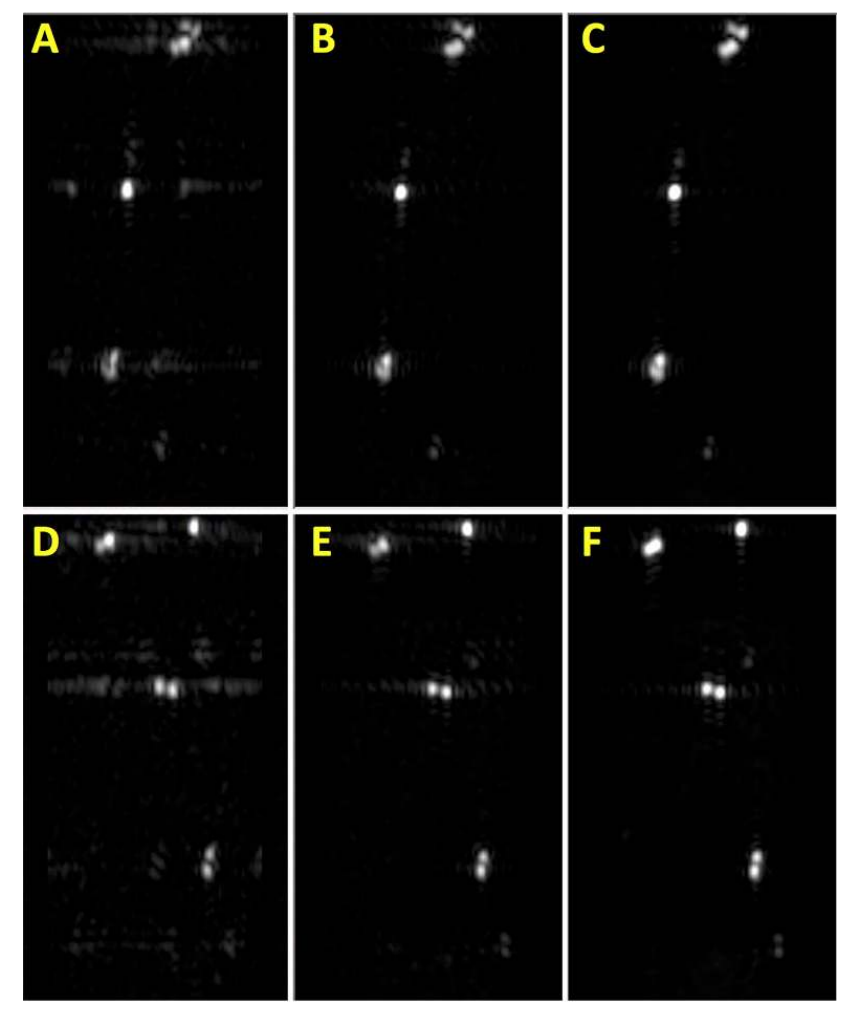

Images 2D obtenues à 4,7 T avec la séquence FLASH 2D (S2): coupes sagittales (A-B-C) et coronale (D-E-F) obtenues avec : A-D Nacc = 1 (durée $96 \mathrm{~ms}$ ); B-E : Nacc = 12 (durée $2 \mathrm{~s}$ ) C-F Nacc = 128 (durée $48 \mathrm{~s}$ ). Nacc = Nombre d'accumulations de la même séquence.

(c) M. Dojat, H. Mathieu, Inserm, 2018. 


\section{(fig. 5 , fig. 6) pour la détection des larves en un temps acceptable (10 min $14 \mathrm{~s})$ pour nos} trois valeurs de champs.

30

31

Table 3

Valeurs obtenues avec la séquence S3 (FLASH 3D, résolution $1 \times 1 \times 1 \mathrm{~mm}^{3}$ ). Nacc : Nombre d'accumulations de la même séquence.

\begin{tabular}{|c|c|c|c|}
\hline & \multicolumn{3}{|c|}{ Imageur } \\
\hline & $4,7 \mathrm{~T}$ & $7 \mathrm{~T}$ & $9,4 \mathrm{~T}$ \\
\hline Nacc (durée) & \multicolumn{3}{|c|}{ Nombre de larves détectées } \\
\hline $1(2 s)$ & $4-5$ & 7 & 7 \\
\hline $20(48 s)$ & $6-7$ & $6-7$ & 7 \\
\hline $256(10 \min 14 \mathrm{~s})$ & 7 & 7 & 7 \\
\hline
\end{tabular}

Figure 5

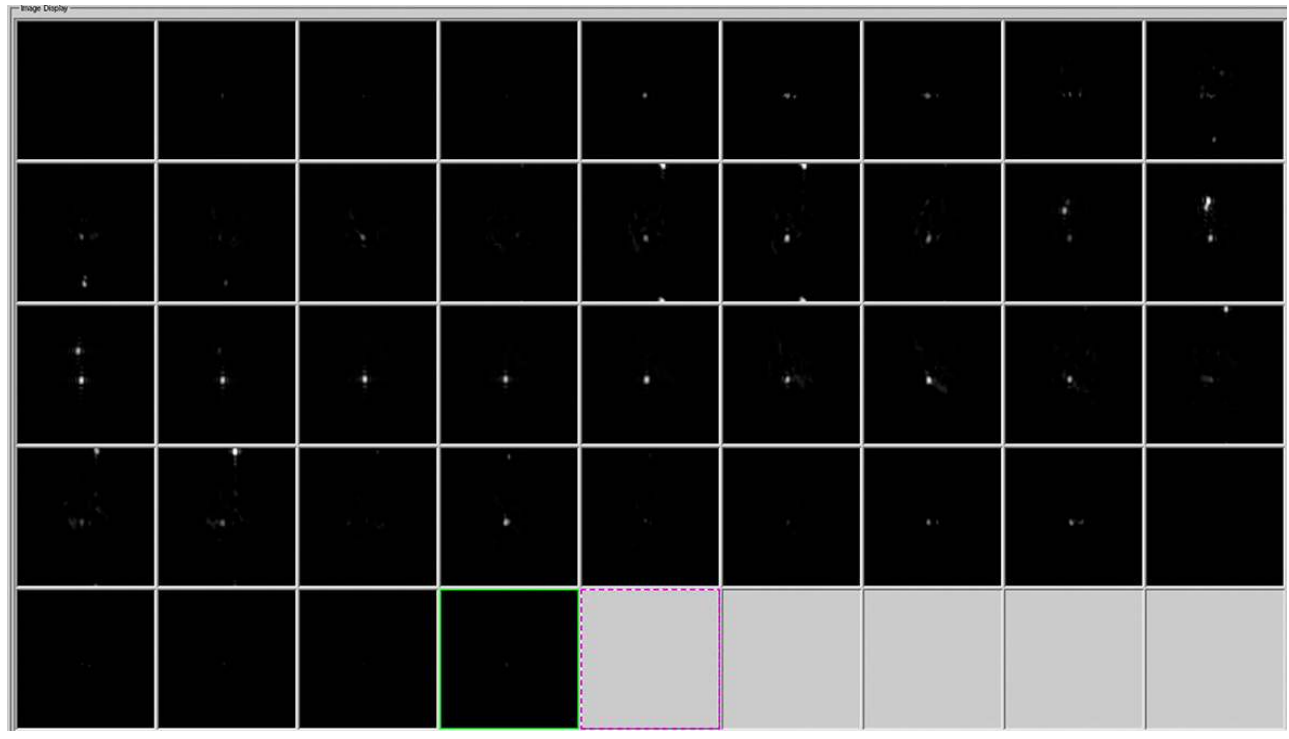

Images 2D obtenues avec la séquence FLASH 3D (S3) à 4,7 T, Nacc $=256$. Chaque image représente une coupe coronale avec un champ de vue de $40 \times 80 \mathrm{~mm}^{2}$. Une épaisseur de $40 \mathrm{~mm}$ de bois a été explorée, soit une image tous les $1 \mathrm{~mm}$. Chaque tache blanche représente un pixel contenant une larve. Nacc $=$ Nombre d'accumulations de la même séquence.

(c) M. Dojat, H. Mathieu, Inserm, 2018. 
Figure 6
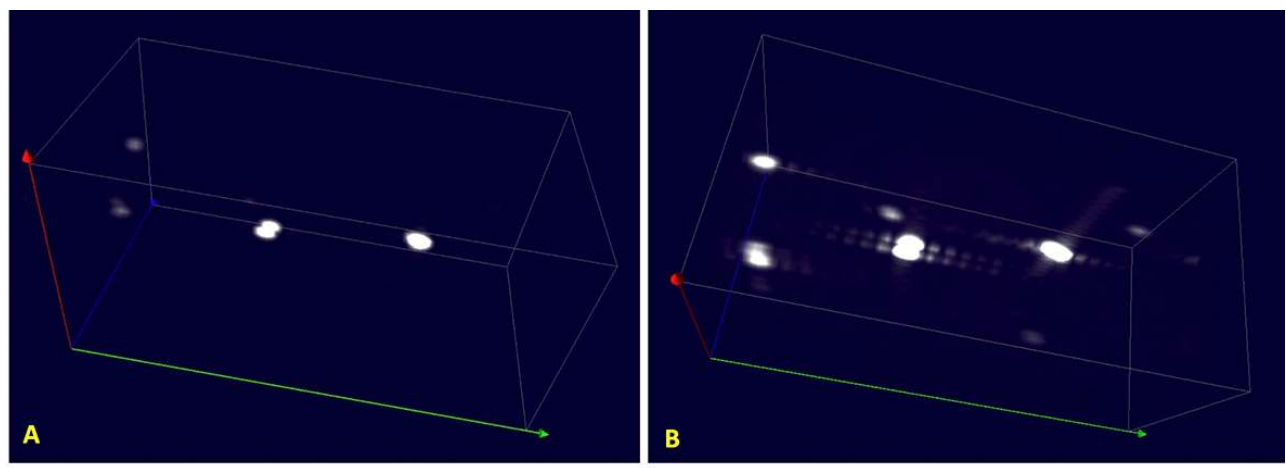

Représentation 3D (Maximum Intensity Projection) avec la séquence FLASH 3D (S3) à 4,7 T, A : Nacc =

1 (durée $2 \mathrm{~s}$ ) et B : Nacc $=128$ (durée $10 \mathrm{~min} 14 \mathrm{~s}$ ). Sur la figure B sont visibles des artefacts de

"Gibbs », classiques en IRM lorsque la taille des pixels est mal adaptée à la taille de l'objet à imager.

Nacc $=$ Nombre d'accumulations de la même séquence.

(c) M. Dojat, H. Mathieu, Inserm, 2018.

La séquence S4, de résolution plus fine (pixel de $0,54 \times 0,54 \times 0,54 \mathrm{~mm}^{3}$ ) permet la détection des larves avec un meilleur rapport contraste sur bruit (voir fig. 6 vs fig. 7). Une durée d'acquisition plus importante (Nacc > 64) devrait permettre d'obtenir le même résultat à 4,7T qu'à 9,4T.

Table 4

34

Valeurs obtenues avec la séquence S4, (FLASH 3D, résolution $0,54 \times 0,54 \times 0,54 \mathrm{~mm}^{3}$ ). Nacc : Nombre d'accumulations de la même séquence.

\begin{tabular}{|c|c|c|c|}
\hline & \multicolumn{3}{|c|}{ Imageur } \\
\hline & $4,7 \mathrm{~T}$ & $7 \mathrm{~T}$ & $9,4 \mathrm{~T}$ \\
\hline Nacc (durée) & \multicolumn{3}{|c|}{ Nombre de larves détectées } \\
\hline $1(9 \mathrm{~s})$ & 6 & 4 & 6 \\
\hline $20(3 \min 12 \mathrm{~s})$ & 6 & 5 & 7 \\
\hline $64(10 \min 14 s)$ & 6 & 5 & 7 \\
\hline
\end{tabular}




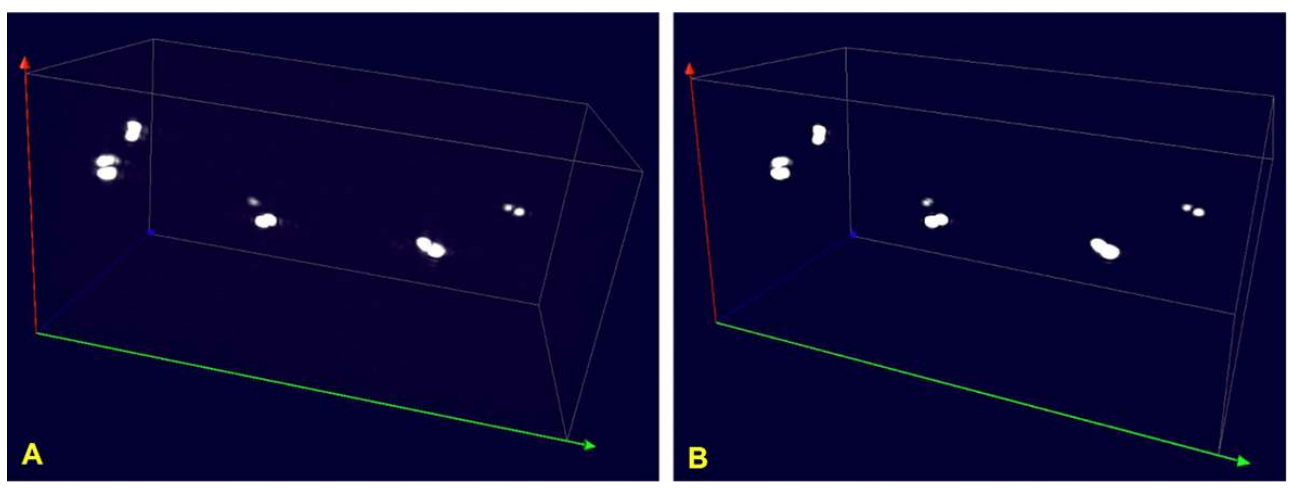

Représentation 3D (Maximum Intensity Projection) avec la séquence FLASH 3D (S4) à 4,7 T, A : Nacc = 1 (durée $9 \mathrm{~s}$ ) et $\mathrm{B}:$ Nacc $=64$ (durée $10 \min 14 \mathrm{~s}$ ). Nacc $=$ Nombre d'accumulations de la même séquence.

(c) M. Dojat, H. Mathieu, Inserm, 2018.

Le nombre de larves détectées par les séquences $\mathrm{S} 3$ et $\mathrm{S} 4$ est légèrement différent. Seule la connaissance de la vérité terrain (nombre réel de larves dans le bois) permettra de choisir la plus optimale.

Nous retiendrons de cette étude qu'un imageur à 4,7 T permet la détection fiable des larves pour des pièces ayant un diamètre jusqu'à $300 \mathrm{~mm}$, avec le protocole suivant :

1 - Repérage du bois : séquence $\mathrm{S} 1, \mathrm{Nacc}=1$, durée $2 \mathrm{~s}$.

2 - Détection des larves : séquence $\mathrm{S} 2, \mathrm{Nacc}=128$, durée $12 \mathrm{~s}$.

3 - Localisation et comptage des larves : séquence S4 (ou S3), Nacc = 64 (ou 256),

durée $10 \min 14 \mathrm{~s}$.

\section{Détection de larves de capricornes}

41 Ces séquences ont également été utilisées pour détecter la présence de larves dans des échantillons de bois préalablement infestés (ou non) par des larves de capricornes. À 9,4 T la présence de larves a été détectée dans 6 échantillons sur 8. En se basant sur l'observation des échantillons a posteriori, galeries ou larve visibles (fig. $8 \mathrm{~A}$ ), échantillon réduit en poudre par les larves ou présence d'un insecte formé (fig. 8 B), cette technique se révèle d'une efficacité de détection de $100 \%$. La Figure 9 illustre la possibilité de voir la larve en mouvement. En outre, une méthode à temps d'écho très court (fig. 9), technique Ultra short TE imaging (UTE 3D) permettant de visualiser le bois, a été testée (paramètres de la séquence : TR/TE : $5 \mathrm{~ms} / 0,011 \mathrm{~ms}$, angle d'excitation $10^{\circ}$, durée : 3 min $11 \mathrm{~s}$, champ de vue $58 \times 58 \times 58 \mathrm{~mm}^{3}$ et taille de pixel $0,625 \mathrm{~mm}$ x 0,625 mm,). On peut alors envisager une quantification fine de la perte de bois et la localisation des galeries (fig. 10). 
Figure 8
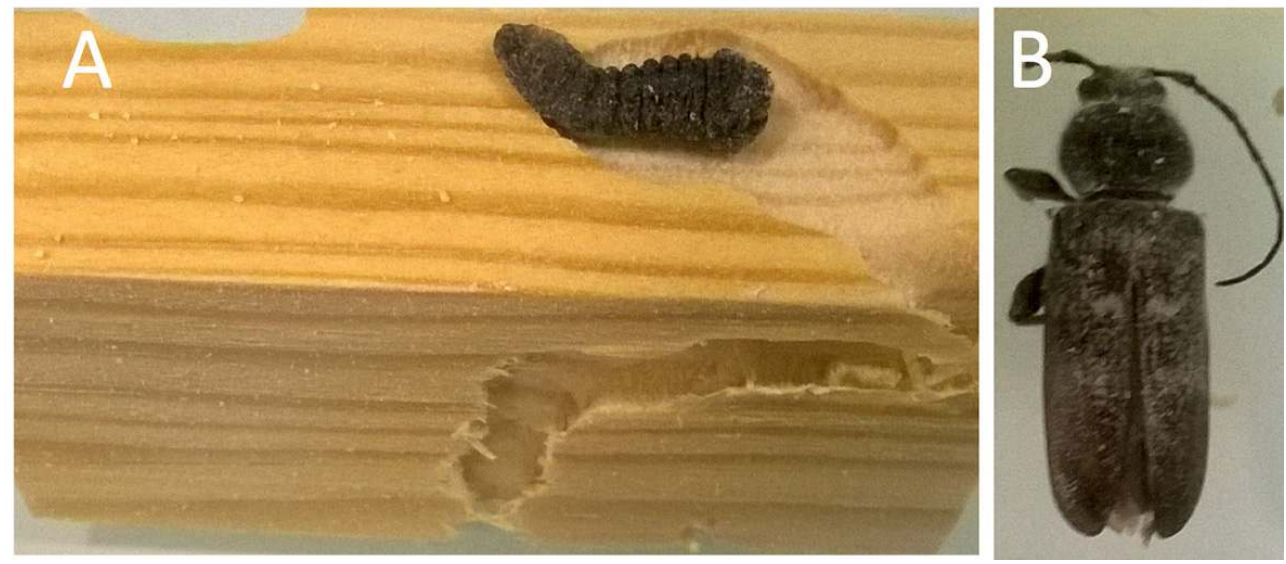

A : pièce de bois contenant une larve de capricorne. B : capricorne adulte.

(c) M. Dojat, H. Mathieu, Inserm, 2018

Figure 9
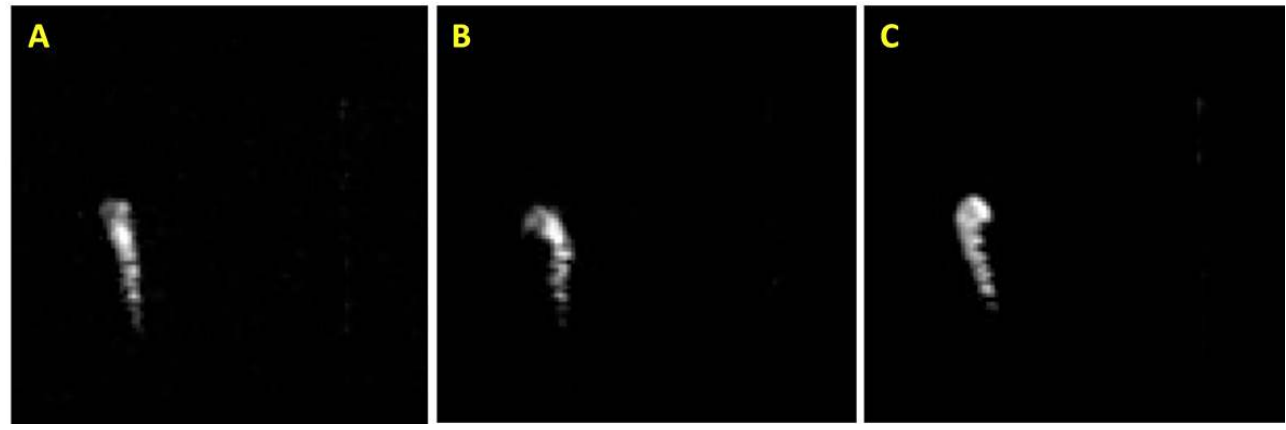

A-B-C : Images d'une même larve de capricorne prise successivement à intervalles de 10 s. Le mouvement de la larve est bien perceptible.

(c) M. Dojat, H. Mathieu, Inserm, 2018.

\section{Figure 10}
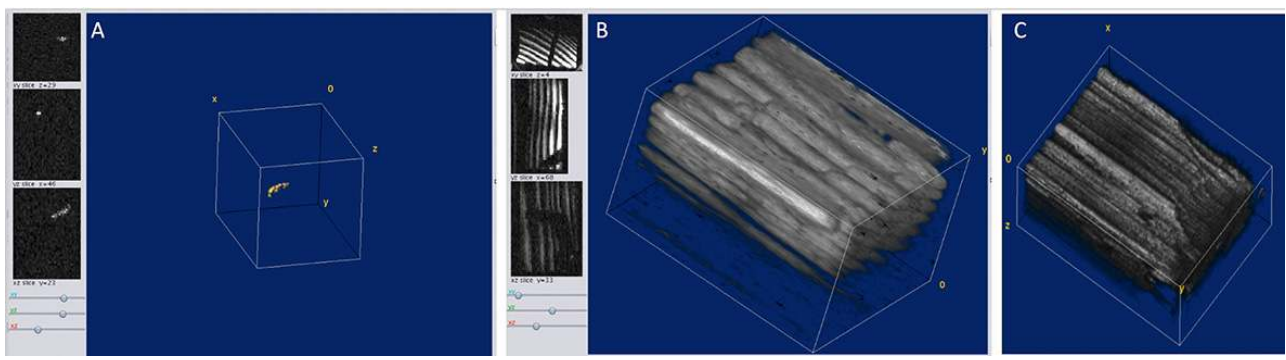

Image sur un échantillon de bois contenant des larves de capricorne à 9,4 T. A : Reconstitution tridimensionnelle de la larve à partir des images de coupes à gauche (séquence FLASH 3D, Nacc : 12). $\mathrm{B}$ : Reconstitution tridimensionnelle de l'échantillon de bois à partir des images de coupes à gauche (séquence UTE). Les galeries sont visibles en noir, parallèles aux strates de bois (gauche). C : La réduction de la masse de bois bien visible.

(c) M. Dojat, H. Mathieu, Inserm, 2018. 


\section{Discussion}

Dans cette étude, nous montrons le riche potentiel de la technique d'imagerie par résonance magnétique (IRM) pour la détection et le suivi d'infestations dans les biens culturels. Peu de travaux ont exploré l'usage de l'IRM pour la détection d'insectes. Il s'agit principalement d'une utilisation pour le suivi d'infestations dans les produits alimentaires $(7,8)$. Pour distinguer rapidement les fruits sains des fruits infestés par la larve Carposina sasakii, Haishi et coll. ${ }^{9}$ construisent un imageur à bas champ (0.2 T) spécifiquement pour cette application. Bao et coll. ${ }^{10}$ utilisent un imageur standard 4,7 T pour détecter et quantifier des larves (Anisakis simplex) parasites des mammifères marins. Mietchen et coll. ${ }^{11}$ utilisent l'IRM et l'analyse spectroscopique à $9,4 \mathrm{~T}$ pour suivre les larves epiblema scudderiana et eurosta solidaginis à basse température $\left(-70^{\circ} \mathrm{C}-0^{\circ} \mathrm{C}\right)$. À notre connaissance, notre travail représente la première tentative exploratoire de détection d'insectes dans des pièces en bois par IRM. Comme la méthode acoustique, cette technique permet de détecter rapidement $(3-6 \mathrm{sec})$, dans des biens organiques ne contenant pas de métal, la présence de larves de petite taille comme Anobium Punctatum (1-2 mm) et de capricorne des maisons, Hylotrupes bajulus, plus grosses (1-2 cm, voir fig. 8 ). De plus, elle permet d'imager les larves en mouvement (voir fig. 9). Contrairement à la méthode par émission acoustique, les galeries creusées dans les matériaux peuvent être qualitativement visualisées (voir fig. 10). Des opérateurs standard de traitement d'images peuvent permettre une quantification automatique du nombre de larves présentes par tranche volumique de pièce et d'estimer la perte en matériau. Ces dernières données permettent d'appréhender objectivement la perte de cohésion des biens culturels et d'adapter le traitement en conséquence.

Noter que l'utilisation d'un champ plus intense n'est pas ici le gage d'une meilleure détection. Si le rapport signal sur bruit est intrinsèquement plus important à $9.4 \mathrm{~T}$, comparativement à $7 \mathrm{~T}$ et $4,7 \mathrm{~T}$, les temps de relaxation des différents composants des échantillons (bois-larve) ne sont pas identiques aux différents champs. Il faudrait donc optimiser finement les paramètres des séquences en fonction du champ pour espérer maximiser la détection. Dans cette étude, les paramètres ont été maintenus constants pour chaque séquence à différents champs. De plus, l'hétérogénéité intrinsèque de nos échantillons interdit l'utilisation d'un réglage automatique (magnetic shimming) afin d'homogénéiser le champ magnétique Bo sur l'ensemble de l'échantillon et donc optimiser la qualité du signal mesuré, donc la détection. Cette étape d'homogénéisation est d'autant plus nécessaire que la valeur de champ est élevée. Un aimant de diamètre interne plus important $(400 \mathrm{~mm}$ à $4,7 \mathrm{~T}$ contre $200 \mathrm{~mm}$ à $7 \mathrm{~T}$ et $9,4 \mathrm{~T})$ aura intrinsèquement un champ plus homogène, favorisant ainsi la qualité de signal pour le champ le moins intense.

Ce travail mérite d'être poursuivi sur trois axes. Les résultats obtenus à $4,7 \mathrm{~T}$ permettent d'envisager l'utilisation d'imageurs de type clinique à plus bas champ ( $3 \mathrm{~T}$ ou $1,5 \mathrm{~T})$, de diamètres internes bien supérieurs $(500 \mathrm{à} 600 \mathrm{~mm})$ afin d'imager des objets de taille importante, levant ainsi une limitation de la micro-tomographie, avec un meilleur contraste que le scanner X pour les tissus mous. Une étude comparative pourrait être menée avec une méthode acoustique émergente (3), pour définir les spécificités et les domaines applicatifs de chacune sur différents types d'infestations. Enfin, de nouvelles techniques d'acquisition IRM à temps d'écho très court (par exemple de type UTE/ZTE), 
permettant d'obtenir un bon signal dans le bois, devront être introduites pour faciliter la quantification de la perte en bois et la détection des larves de plus petite taille.

\section{NOTES}

1. - FLEMING, M., BHARDWAJ, M., JANOWIAK, J., SHIELD, J., ROY, R., AGRAWAL, D., et al. " Noncontact ultrasound detection of exotic insects in wood packing materials ». Forest products journal, 2005, 55(6), p. 33-37.

2. - WATANABE, H., YANASE, Y., \& FUJII, Y. "Relationship between the movements of the mouthparts of the bamboo powder-post beetle Dinoderus minutus and the generation of acoustic emission ». Journal of Wood Science, 2016, 62, p. 85-92.

3. - LE CONTE, S., VAIEDELICH, S., THOMAS, J.-H., MULIAVA, Va., DE REYER, D., \& MAURIN, E. "Acoustic emission to detect xylophagous insects in wooden musical instrument ». Journal of Cultural Heritage, 16(3), p. 338-343.

4. - Voir le brevet sur : https://doi.org/10.13140/rg.2.1.4886.4160 [consulté le 14/03/2019].

5. - HALL, MJ., SIMONSEN, TJ., MARTIN-VEGA, D. « The "dance" of life: visualizing metamorphosis during pupation in the blow fly Calliphora vicina by X-ray video imaging and micro-computed tomography ». R Soc Open Sci., 2017, 4(1), p. 1-8, doi/10.1098/rsos.160699.

6. - WATANABE, H., YANASE, Y., \& FUJII, Y. «Evaluation of larval growth process and bamboo consumption of the bamboo powder-post beetle Dinoderus minutus using X-ray computed tomography ». Journal of Wood Science, 2015, 61, p. 171-177.

7. - ARENDSE, E., FAWOLE, OA., MAGWAZA, LS., \& OPARA, UL. «Estimation of the density of pomegranate fruit and their fractions using X-ray computed tomography calibrated with polymeric materials ». Biosystems Engineering, 2016, 148, p. 148-156.

8. - Voir les sites: https://www.imaios.com/fr/e-Cours/e-MRI ou http://www.cis.rit.edu/ htbooks/mri/inside.htm pour un tutoriel détaillé sur les bases de l'IRM [consultés le 13/03/2019]. 9. - HAISHI, T., KOIZUMI, H., ARAI, T., KOIZUMI, M., KANO, H. « Rapid Detection of Infestation of Apple Fruits by the Peach Fruit Moth, Carposina sasakii Matsumura, Larvae Using a 0.2-T Dedicated Magnetic Resonance Imaging Apparatus ». Appl Magn Reson., 2011, 41(1), p. 1-18.

10. - BAO, M., STRACHAN, NJC., HASTIE, LC., MACKENZIE, K., SETON, HC., PIERCE, GJ. « Employing visual inspection and Magnetic Resonance Imaging to investigate Anisakis simplex s.l. infection in herring viscera ». Food Control, 2017, 75, p. 40-47.

11. - MIETCHEN, D., MANZ, B., VOLKE, F., \& STOREY, K. « In vivo assessment of cold adaptation in insect larvae by magnetic resonance imaging and magnetic resonance spectroscopy ». PLoS One, 2008, 3(12), e3826. 


\section{RÉSUMÉS}

Nous proposons d'utiliser l'imagerie par résonance magnétique (IRM) pour détecter et quantifier les larves d'insectes nuisibles dans les biens culturels en bois et plus généralement, constitués de matériaux organiques. Nous rapportons les résultats de différentes expériences réalisées à différents niveaux de champ magnétique pour détecter la présence d'insectes xylophages dans des pièces en bois. Nous démontrons l'intérêt de cette technique non invasive pour la détection des infestations par des larves de petite taille, telle la petite vrillette (Anobium punctatum) ou plus grosses, tel le capricorne des maisons (Hylotrupes bajulus). Différentes pistes sont détaillées qui restent à explorer pour son utilisation dans le domaine patrimonial.

The infestation of a work of art may be destructive for the piece itself and also presents the threat of spreading throughout an entire collection. Several methods are available to museums in order to diagnose an infestation at an early stage and then treat it and prevent possible proliferation. Imaging offers a large range of non-destructive techniques capable of detecting the presence of larvae and insect eggs in work of art. In this study we explore the use of the techniques of magnetic resonance imaging (MRI) for the detection in vivo and quantification of xylophagous larvae in wood. Our results were obtained using different levels of magnetic fields for the detection of the small larvae of common furniture beetles and the larger larvae of longhorn beetles. Our study demonstrates the interest of MRI techniques for the non-destructive detection of infestation in works of art made of wood and other organic materials, techniques that can be used by museum curators.

\section{INDEX}

Keywords : non-destructive control, infestation, insect, xylophage, cultural property, magnetic field, preventive conservation, infestation detection, IPM (integrated pest management), heritage pests

Mots-clés : contrôle non destructif, infestation, insecte, xylophage, bien culturel, champ magnétique, conservation préventive, détection des infestations, IPM (integrated pest management), nuisible du patrimoine

\section{AUTEURS}

\section{GRAZIA NICOSIA}

Conservateur-restaurateur, conseil en conservation préventive, service de la Conservation préventive, direction de la Recherche et des Collections, musée du Louvre Grazia.Nicosia@louvre.fr

\section{HERVÉ MATHIEU}

Ingénieur de recherche, CNRS, UMR 3552, Grenoble 


\section{JOËLLE LE ROUX}

Conservateur-restaurateur, conseil en conservation préventive, service de la Conservation préventive, direction de la Recherche et des Collections, musée du Louvre Joelle.LeRoux@louvre.fr

\section{ANNE DE WALLENS}

Chef du service de la Conservation préventive, direction de la Recherche et des Collections, musée du Louvre Anne.De-Wallens@louvre.fr

\section{MICHEL DOJAT}

Directeur de recherche, Université Grenoble Alpes, Inserm, CHU Grenoble Alpes, GIN Michel.Dojat@univ-grenoble-alpes.fr 